\title{
RESEARCH
}

Open Access

\section{Value of diffusion tensor imaging and tractography in unilateral lumbar disc prolapse}

\author{
Ahmed Sayed Abd El Basset ${ }^{1 *}$ (D), Ahmed Hesham Mohamed Saeed', Mona Hussein Tawfik ${ }^{2}$ and \\ Marwa Shehata Abd El Hady ${ }^{1}$
}

\begin{abstract}
Background: Conventional MR imaging is not enough for evaluation of symptomatic foraminal stenosis, because there is high incidence of false-positive results in asymptomatic elderly patients. Conventional MR cannot quantitatively assess the severity of the nerve lesion. DTI is a non-invasive way to effectively trace the nerve fiber bundle and quantitatively evaluate the nerve injury. DTI with fiber tracking may describe abnormalities beyond the resolution of conventional MR techniques. The aim of this work was to compare between the mean values of diffusion parameters such as fractional anisotropy and apparent diffusion coefficient of the compressed lumbar spinal nerve roots and of the contralateral normal nerve roots. Correlate these parameters with the severity of neurological symptoms. This is a prospective study that was conducted on 50 patients with symptomatic unilateral posterolateral lumbar disc prolapse at a university hospital. They were investigated with diffusion tensor imaging with tractography on a 1.5-T MR. The changes in the mean fractional anisotropy and apparent diffusion coefficient values of the compressed nerves and the relationship between these changes and the severity of the neurological side effects using Japanese Orthopedic Association score and visual analogue scale were investigated.
\end{abstract}

Results: The mean fractional anisotropy values were significantly lower ( $p \leq 0.001)$, and mean apparent diffusion coefficient values were significantly higher $(p \leq 0.001)$ in compressed nerves than in contralateral intact nerves. There were strong correlations between the DTI parameters and the severity of the neurological symptoms as assessed using the Japanese Orthopedic Association score and the visual analogue scale.

Conclusion: In patients with lumbar disc prolapse, radicular diffusion parameters are affected in the compressed roots in comparison to the healthy roots and this affection is correlated with the degree of prolapse and with the severity of neurological symptoms. DTI with fiber tracking provide clinically relevant information and describe abnormalities beyond the resolution of conventional MR techniques.

Keywords: Lumbar disc prolapse, Diffusion tensor imaging, Fiber tractography, Fractional anisotropy (FA), Apparent diffusion coefficient (ADC), The Japanese Orthopedic Association (JOA) score, Visual analog scale (VAS)

\footnotetext{
* Correspondence: dr.ahmedbasset@gmail.com

${ }^{1}$ Radiology Department, Faculty of Medicine, Beni-Suef University, Beni Suef,

Egypt

Full list of author information is available at the end of the article
}

(c) The Author(s). 2021 Open Access This article is licensed under a Creative Commons Attribution 4.0 International License, which permits use, sharing, adaptation, distribution and reproduction in any medium or format, as long as you give appropriate credit to the original author(s) and the source, provide a link to the Creative Commons licence, and indicate if changes were made. The images or other third party material in this article are included in the article's Creative Commons licence, unless indicated otherwise in a credit line to the material. If material is not included in the article's Creative Commons licence and your intended use is not permitted by statutory regulation or exceeds the permitted use, you will need to obtain permission directly from the copyright holder. To view a copy of this licence, visit http://creativecommons.org/licenses/by/4.0/. 


\section{Background}

Disc degeneration can be associated with sciatica, lower back pain, and disc herniation or prolapse [1].

Conventional MR imaging is not enough for evaluation of symptomatic foraminal stenosis; DTI with fiber tracking may provide clinically relevant information and may describe abnormalities beyond the resolution of conventional MR techniques. Mean fractional anisotropy (FA) and apparent diffusion coefficient (ADC) map values which reflect microstructural changes have been reported to be abnormal in areas that may appear normal on anatomic MR images [2]. Diffusion tensor imaging (DTI) is an MR technique developed on the basis of the diffusion-weighted imaging (DWI). DTI collects attenuated signal intensity induced by a diffusion of water molecules in all directions of space through a diffusion-sensitive gradient in numerous directions, quantitatively describes the three-dimensional trajectory of spatial diffusion, and can provide a diffusion direction characteristic of living tissue [3].

The main purpose of tractography is to illustrate the orientational architecture of tissues by combining pathways of maximum diffusion coherence. The fibers described with tractography are often considered to represent individual axons or nerve fibers, but more specifically, in physical terms, they are viewed as lines that follow the maximum diffusion and that only generally reflect the axonal architecture [4].

The aim of this work was to compare between the mean values of diffusion parameters such as fractional anisotropy and apparent diffusion coefficient of the compressed lumbar spinal nerve roots and of the contralateral normal nerve roots. Correlate these parameters with the severity of neurological symptoms. DTI with fiber tracking may provide clinically relevant information and may describe abnormalities beyond the resolution of conventional MR techniques.

\section{Methods}

\section{Patient population}

This prospective study included 50 patients; thirtyone men and nineteen women ranged from 24 to 65 years with a mean value $46.72 \pm 12.95$ years who suffered from unilateral radiculopathy and lumbar disc prolapse. All patients underwent DTI scanning. The patients gave informed consent, and the study had prior approval from the faculty of medicine ethics committee. The diagnosis was based on neurological symptoms and MRI images. The location of the symptomatic nerves in the 50 patients was seven at $\mathrm{L} 3 / 4$, thirty-one at L4/5, and twelve at L5/S1.

\section{Excluded patients}

Excluded patients were those with multi-level disc lesions, spinal deformities or spinal canal stenosis, previous history of spinal trauma, previous spinal surgery, and radiological evidence of any inflammatory or neoplastic lesion affecting the spinal cord or vertebral column and patients with contraindications for MRI examination.

\section{Clinical assessment}

Neurological severity was assessed using a visual analogue scale (VAS) for low back pain and leg pain from 10 (extreme pain) to zero (no pain), and the Japanese Orthopedic Association (JOA; 0 to 17 points) scoring system. The normal JOA score is 17 points, based on upper extremity motor function, lower extremity motor function, sensory, bladder, and bowel function.

\section{MRI protocol}

A 1.5-T MRI scanner Siemens MAGNETOM Aera was used in this study. Sagittal T1-weighted (TR/TE, 500/9), axial, and sagittal T2-weighted fast spin-echo (TR/TE, $3000 / 123)$ sequences were obtained using a $248 \times 267$ matrix, 300-mm field of view (FOV), and 4-mm slice thickness/gap.

The DTI series were acquired using spectral presaturation with inversion recovery (SPIR) and an echoplanar imaging (EPI) sequence with a free-breathing scanning technique. Patients were scanned in a supine position using a SENSE Spine-coil. The following imaging parameters were set: $900 \mathrm{~s} / \mathrm{mm}^{2} b$ value, MPG: 15 directions, $6200 / 142 \mathrm{~ms}$ for TR/TE respectively, axial slice orientation, 2-mm slice thickness/gap, 320 $\times 213 \mathrm{~mm}$ field of view (FOV), $96 \times 192$ matrix, 1.2 $\times 1.2 \times 2.0 \mathrm{~mm} 3$ voxel size, four excitations, 50 total slices, 12 min scan time.

\section{Image analysis}

The diffusion tensor was calculated using the regions of interest (ROIs) placed at three levels at 3-mm intervals distal to the site of compression of the nerve by the disc prolapse and on the contralateral healthy nerves on the coronal image of ADC and FA maps and their mean values were calculated. The size of the ROIs was selected to be as precise as possible on the respective nerve roots to avoid partial volume effects when the mean FA and ADC were calculated.

Data was transferred to Siemens work station and processed to perform tractography images.

\section{Statistical analysis}

The data were coded and entered using the statistical package for social science version 18 (SPSS v 18). 
Table 1 Diffusion tensor imaging findings in the included patients

\begin{tabular}{llll}
\hline DTI parameters & $\begin{array}{l}\text { Influenced side } \\
\text { [mean (SD)] }\end{array}$ & $\begin{array}{l}\text { Healthy side } \\
{[\text { mean (SD)] }}\end{array}$ & $\boldsymbol{P}$ value \\
\hline FA & $0.238(0.05)$ & $0.309(0.07)$ & $0.001^{*}$ \\
ADC & $1.466(0.256)$ & $1.256(0.215)$ & $0.001^{*}$ \\
\hline
\end{tabular}

${ }^{*} p$ value $<0.05$ (significant)

Descriptive statistics were reported as mean \pm SD and number (\%) for categorical variables. Student's $t$ test was used for comparison between means of two unpaired groups of quantitative variables. The one way of variance analysis (ANOVA) was used to assess statistical differences of quantitative data between study groups. Bonferroni test was used for groups' multiple comparisons. Chi-squared test was used for comparison between two groups of categorical data. The Pearson correlation coefficient $(r)$ was used to describe the degree of relationship between two variables. The sign of correlation coefficient $(+,-)$ defines the direction of the relationship, either positive or negative. The probability/significance value $(P$ value $) \geq 0.05$ is not statistically significant and $<0.05$ is statistically significant.

\section{Results}

The mean FA value measured $0.238(0.05)$ in compressed nerves compared to $0.309(0.07)$ in the contralateral healthy nerves yet the mean apparent diffusion coefficient value measured $1.466(0.256)$ in compressed nerves compared to $1.256(0.215)$ in the contralateral healthy nerves (Table 1 ).

Patients with disc extrusion had significantly lower mean FA values and higher mean ADC values than those with disc protrusion and those with disc bulge. Patients with disc protrusion had significantly lower mean FA and higher mean ADC values than those with disc bulge (Fig. 1).

There was a statistically positive correlation between FA values of affected nerve and JOA scores. There was a statistically negative correlation between ADC values of affected nerve and the JOA scores (Table 2, Figs. 2 and 3).

There was a strong negative correlation between FA values of affected nerve and the VAS scores. There was also a strong positive correlation between ADC values of affected nerve and the VAS scores (Table 3, Figs. 4 and 5).

Fiber tractography revealed compression, distortion, or displacement of involved spinal roots in lumbar disc prolapse patients (Fig. 6). Involvement of spinal nerve roots on DTI- fiber tractography is shown in (Table 4).

So the mean fractional anisotropy values were significantly lower $(p=0.001)$, and mean apparent diffusion coefficient values were significantly higher $(p=0.001)$ in compressed nerves than in contralateral intact nerves. There were strong correlations between the DTI parameters and the severity of the neurological symptoms as assessed using the Japanese Orthopedic Association score and the visual analogue scale.

\section{Discussion}

Low back pain is one of the most common health problems among population all over the world [5]. About $75-84 \%$ of the general population suffers from low back pain and $5-10 \%$ of them suffering severe morbidity [6]. Men and women are equally affected, and $50 \%$ of adults and $30 \%$ of adolescents are affected

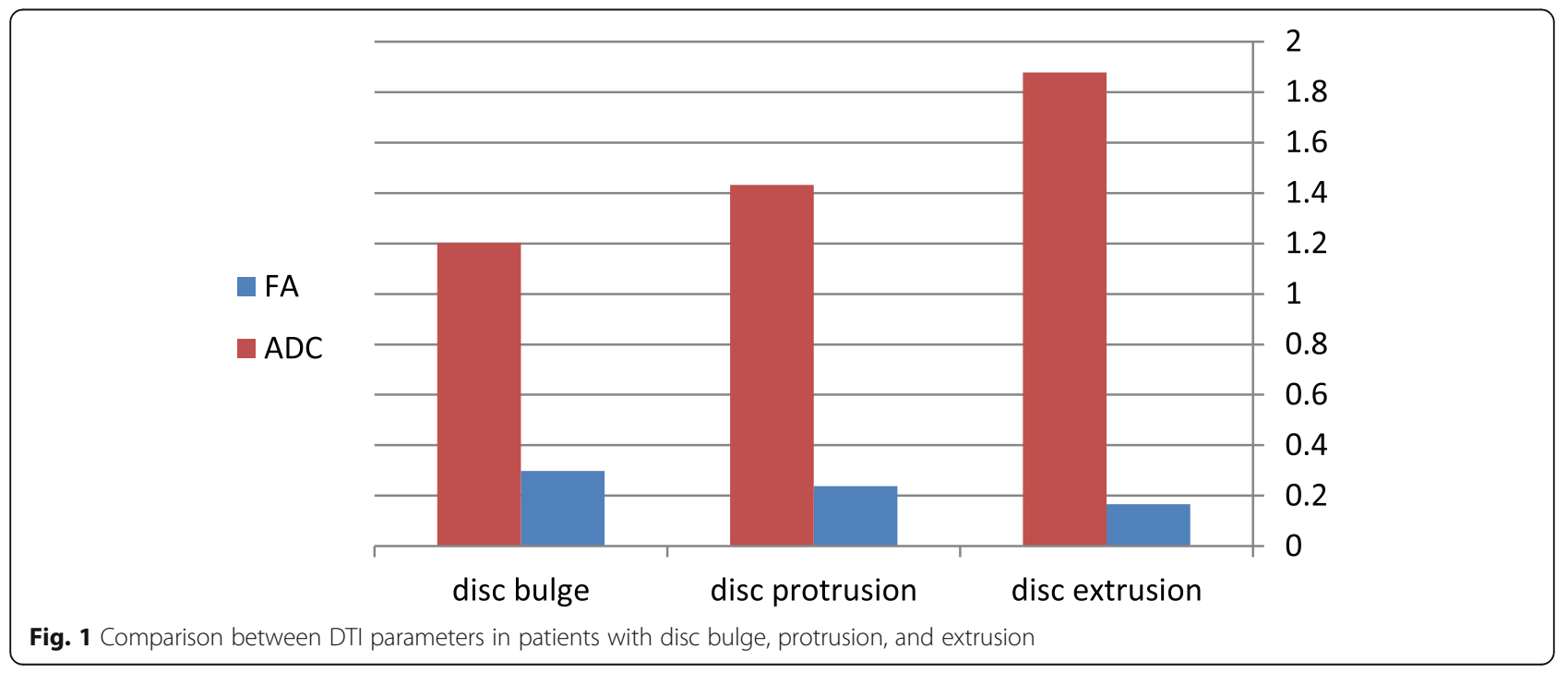


Table 2 Correlation between DTI parameters and JOA scores

\begin{tabular}{lll}
\hline & JOA & \\
\cline { 2 - 3 } & $\boldsymbol{( r ) \text { coef. }}$ & $\boldsymbol{P}$ value \\
\hline FA & 0.4 & $0.004^{*}$ \\
ADC & -0.342 & $0.015^{*}$
\end{tabular}

JOA Japanese Orthopedic Association

${ }^{*} p$ value $<0.05$ (significant)

at least once [7]. Lumbar disc prolapse is considered one of the most common causes for low back pain [8]. Disc prolapse mechanically compresses adjacent nerve roots causing inflammatory changes, leading to sensory manifestations, motor abnormalities or sphincteric troubles [9].

The degree of nerve root compression observed in MRI is often inconsistent with the patient's symptoms. Tractography is to illustrate the orientational architecture of tissues by combining pathways of maximum diffusion coherence [4]. High ADC values are consistent with increase of the extracellular space, usually representing edema regardless of its origin. FA is an index of fiber organization, which reflects the integrity of axonal bundles and the directionality of water movement delimited by physiological barriers such as myelin sheaths, endoneurium, perineurium, or epineurium [10].

Our results were consistent with previous articles from the literature such as Balbi et al. [11], Shi et al. [12], and Zhang et al. [13] and revealed that the mean FA value for the compressed nerve root was significantly lower than that of contralateral healthy nerve root, and the mean ADC value of the affected nerve root was significantly higher than that of contralateral healthy nerve root.

These changes in diffusion parameters might be related to histological changes occurring in the compressed nerve root. As described in experimental studies, chronic compression and chemical irritation of lumbar nerve root lead to variable histological changes, including the acceleration of vascular permeability with disrupted nerve root barrier and development of intraneural edema and hyperemia in and around the nerves. Also, compression induces reduced blood flow and ischemia leading to demyelinated nerve fibers, Wallerian degeneration, and endoneural cracking [14]. These microstructural changes occurring in compressed nerve roots lead to increase diffusion in perpendicular plane to the largest eigenvalue resulting in a decrease in FA, indicating a more isotropic diffusion in the tissue. As for the increase in $\mathrm{ADC}$, we may assume that the intraneural edema and the increased distance in axon fascicle occurring in a compressed nerve root increases the water diffusivity along the nerve bundles. These hypotheses may be corroborated by two previous experimental studies reporting a decreased FA in rat and frog segments of sciatic nerves undergoing Wallerian degeneration [15]. However, due to various factors (i.e., magnetic intensity, scanning parameters, motion and chemical shift artifacts, and ROI differences), the differences of the results in DTI metrics were frequently reported in previous studies as $\mathrm{Wu}$ et al. [16].

Our study also found that patients with disc extrusion had significantly lower mean FA values and higher mean ADC values than those with disc protrusion and those with disc bulge. Patients with disc protrusion had

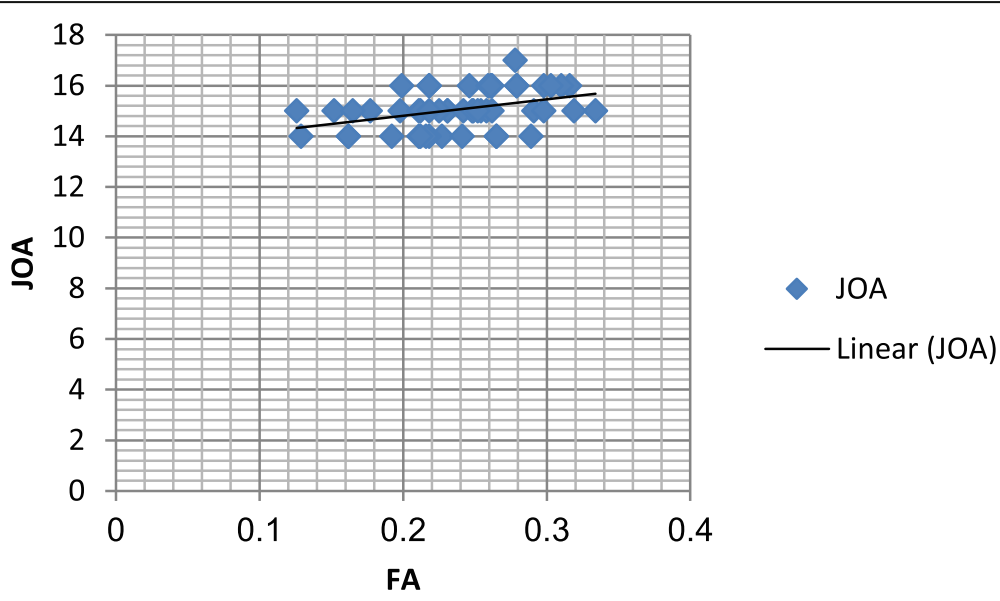

Fig. 2 Correlation between FA values and JOA scores for compressed nerves 


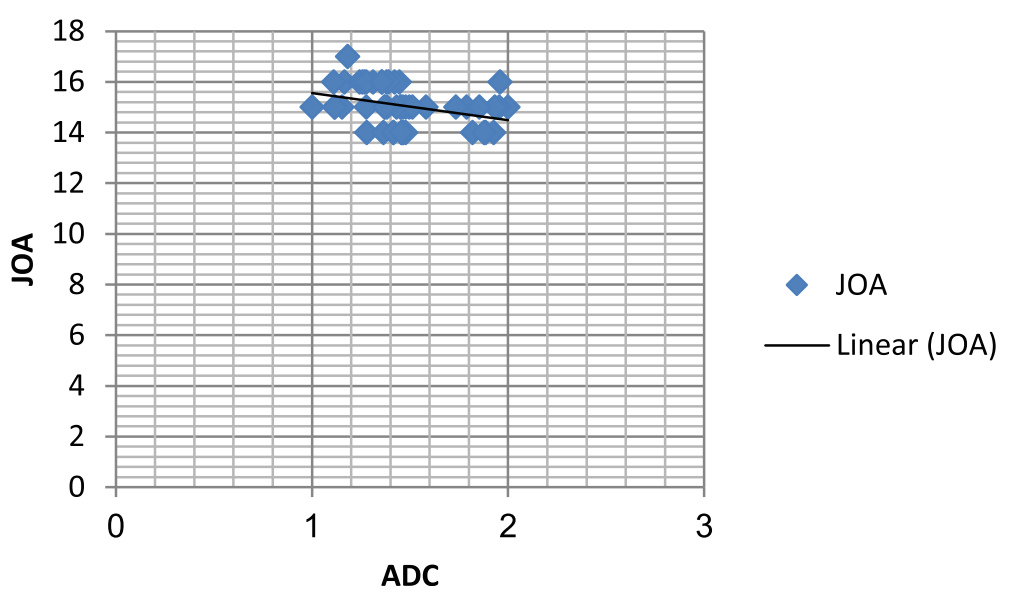

Fig. 3 Correlation between ADC and JOA scores for compressed nerves

significantly lower mean FA and higher mean ADC values than those with disc bulge, which is consistent with other study by Zhang et al. [13].

Our study revealed that there was a statistically positive correlation between FA values of affected nerve and JOA scores. There was a statistically negative correlation between ADC values of affected nerve and the JOA scores, which is consistent with other study by Eguchi et al. [17].

In accordance with other study by Zhang et al. [13], our study revealed that there was a strong negative correlation between FA values of affected nerve and the VAS scores. There was also a strong positive correlation between ADC values of affected nerve and the VAS scores Contrary to our findings, another study by Takashima et al. [18] failed to detect any significant correlation between ADC values and VAS scores. They attributed their findings to the difference in the way of measuring of ADC, using short TI recovery as the DWI fat suppression method or the water-selective excitation technique as the DWI fat suppression method, which yields a higher signal-to-noise ratio than the short TI recovery method.

DTI has a few limitations. Firstly, tractography is numerical modeling of the diffusion tensor data using

Table 3 Correlation between DTI parameters and VAS scores

\begin{tabular}{lll}
\hline & VAS & \\
\cline { 2 - 3 } & $(\boldsymbol{r})$ coef. & $\boldsymbol{P}$ value \\
\hline FA & -0.87 & $0.001^{*}$ \\
ADC & 0.671 & $0.001^{*}$ \\
\hline $\begin{array}{l}\text { VAS visual analogue scale } \\
{ }^{*} p \text { value }<0.05 \text { (significant) }\end{array}$ & &
\end{tabular}

probability theories to model the most likely course of diffusion, and the number of tracts visualized by DTI did not present the real volume of nerve fiber trajectories. Second, the magnetic susceptibility effect and increases in motion artifacts lead to signal irregularities and image distortion so that nerve fiber follow-up is limited in areas with artifacts. Third, the evidence is insufficient to support DTI as a diagnostic tool or predictor of clinical outcomes. Fourth, automatic analysis methods such as tract-specific automatic ROI placement are needed [19].

When patients' symptoms are inconsistent with MRI findings, or when MRI reveals multi-stage disc prolapse, accurate diagnosis can reduce the degree of decompression, limit surgical trauma, and diminish the cost of hospitalization. Recent studies have shown the application of MRI combined with DTI or paraspinal mapping (PM) can reduce false positives on MRI and also replace invasive methods such as discography and selective nerve root block to further enhance diagnosis [20].

\section{Conclusion}

- In patients with symptomatic lumbar disc prolapse, radicular diffusion parameters are affected in the compressed roots in comparison to the healthy roots.

- The affection of radicular diffusion parameters in patients with symptomatic lumbar disc prolapse is correlated with the degree of prolapse.

- This study has limitations. First, the relatively small sample size, meaning a large number of clinical studies is required to confirm repeatability of our results. Second, the cross-sectional area of the nerve 


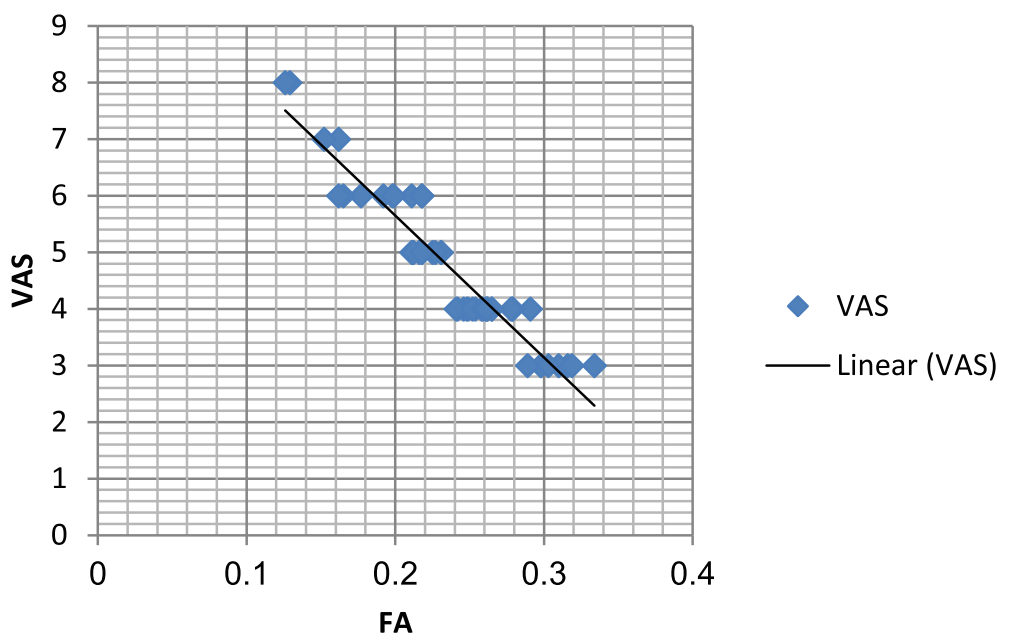

Fig. 4 Correlation between FA and VAS scores for compressed nerves

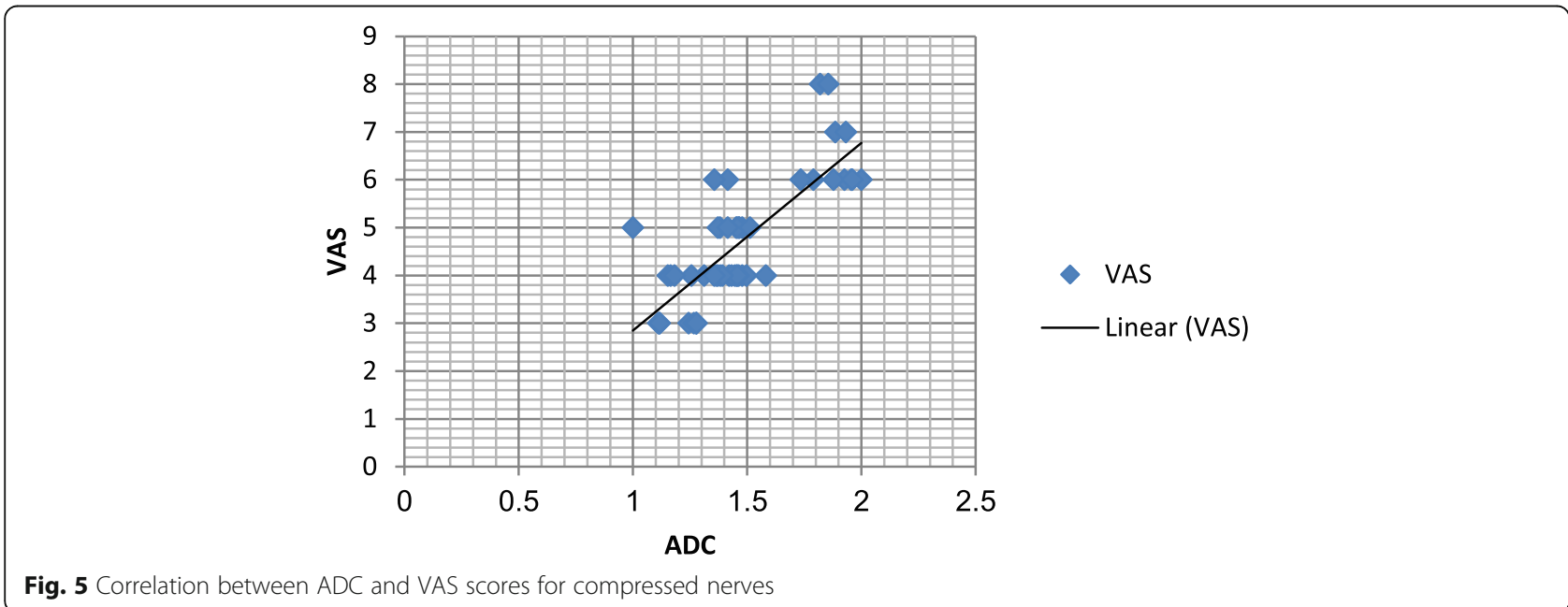

Fig. 5 Correlation between ADC and VAS scores for compressed nerves 


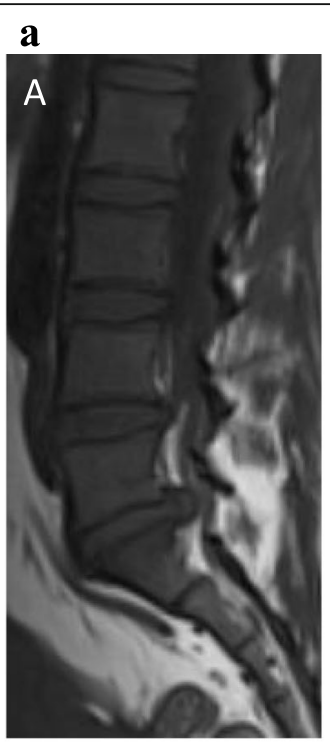

b

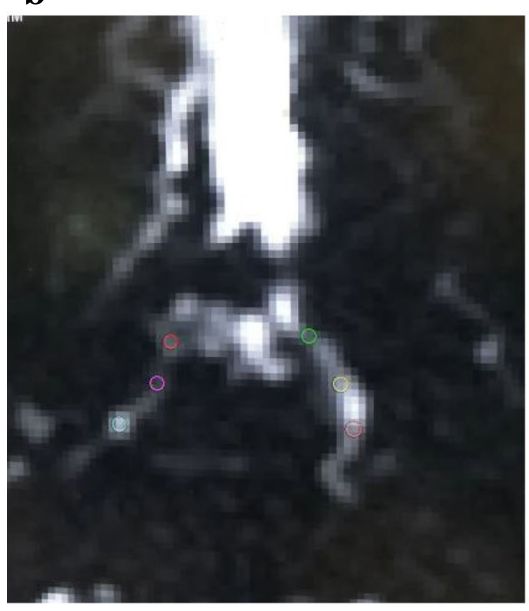

C
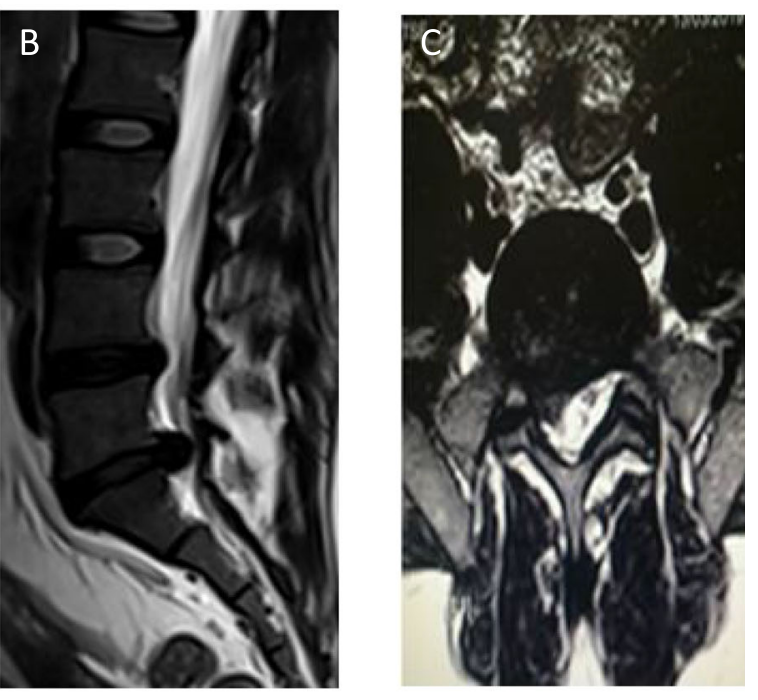

\begin{tabular}{|c|c|c|c|c|}
\hline 10 & $\begin{array}{l}\text { FA } \\
\text { Mean }{ }_{\text {SDer }} \\
\text { Size/Min/Max }\end{array}$ & $\begin{array}{l}\text { ADC } \\
\text { Mean SDer } \\
\text { Size/Min/Max }\end{array}$ & $\begin{array}{c}\text { TraceW } \\
\text { Mean SDev } \\
\text { Size / Min/Max }\end{array}$ & $\begin{array}{c}\text { 60 } \\
\text { Mean SDev } \\
\text { Size } / \text { Min } / \text { Max }\end{array}$ \\
\hline & $\begin{array}{cc}148.7 \quad 23.9 \\
3 / 120 / 178\end{array}$ & $\begin{array}{l}2209.7 \quad 37.6 \\
3 / 2162 / 2254\end{array}$ & $\begin{array}{lr}134.3 & 13.0 \\
3 / 118 / 145\end{array}$ & $\begin{array}{r}1222.7 \quad 122.4 \\
3 / 1057 / 1349\end{array}$ \\
\hline 2 & $\begin{array}{cc}137.8 & 31.7 \\
4 / 87 / 165\end{array}$ & $\begin{array}{lr}2208.0 & 149.5 \\
4 / 2047 / 2397\end{array}$ & $\begin{array}{cr}85.8 & 4.2 \\
4 / 81 / 82\end{array}$ & $\begin{array}{c}788.3 \quad 113.3 \\
4 / 627 / 809\end{array}$ \\
\hline 3 & $\begin{array}{cr}236.0 & 57.1 \\
3 / 182 / 315\end{array}$ & $\begin{array}{rr}1650.7 & 190.2 \\
3 / 1468 / 1913\end{array}$ & $\begin{array}{c}171.3 \quad 12.4 \\
3 / 154 / 182\end{array}$ & $\begin{array}{ll}896.3 & 107.6 \\
3 / 788 / 1043\end{array}$ \\
\hline 4 & $\begin{array}{cr}746.5 & 15.5 \\
2 / 731 / 762\end{array}$ & $\begin{array}{cr}364.5 & 33.5 \\
2 / 331 / 398\end{array}$ & $\begin{array}{cc}73.5 & 5.5 \\
2168 / 79\end{array}$ & $\begin{array}{cc}105.5 & 11.5 \\
2 / 84 / 117\end{array}$ \\
\hline 5 & $\begin{array}{cc}200.3 & 40.3 \\
4 / 148 / 261\end{array}$ & $\begin{array}{l}1442.8 \quad 31.8 \\
4 / 1401 / 1489\end{array}$ & $\begin{array}{l}80.3 \quad 4.4 \\
4 / 64 / 96\end{array}$ & $\begin{array}{cc}383.3 & 27.1 \\
4 / 343 / 410\end{array}$ \\
\hline 6 & $\begin{array}{cc}371.8 & 48.1 \\
4 / 316 / 446\end{array}$ & $\begin{array}{ll}1321.3 & 25.7 \\
4 / 1287 / 1348\end{array}$ & $\begin{array}{cc}77.8 & 7.7 \\
4 / 66 / 86\end{array}$ & $\begin{array}{cr}291.0 \quad 34.4 \\
4 / 242 / 328\end{array}$ \\
\hline
\end{tabular}

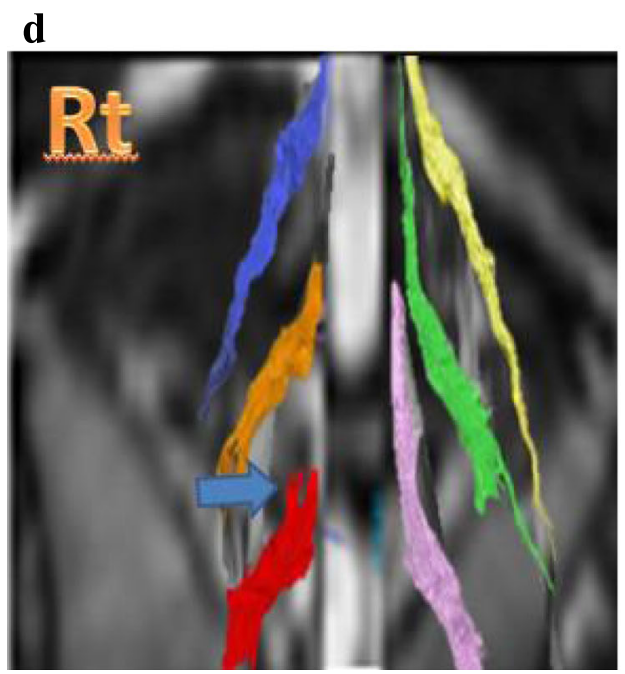

Fig. 6 a Thirty-five-year-old male patient, MRI sagittal T1 (A), sagittal T2 (B), and axial T2 (C) images show L5/S1 right posterolateral disc extrusion. b, c DTI of the same patient with FA and ADC values measured along right and left S1 nerves using ROI revealed mean FA of right nerve 0.194 and of left nerve 0.418. Mean ADC of right nerve 1767 and of left nerve 1297. $\mathbf{d}$ Tractography image of the same patient revealed distortion of right S1 nerve root 
Table 4 Involvement of spinal nerve roots on DTl-fiber tractography

\begin{tabular}{lll}
\hline Nerve roots & Compression and distortion & Displacement \\
\hline L4 & 5 & 2 \\
L5 & 25 & 6 \\
S1 & 8 & 4 \\
\hline
\end{tabular}

root was minute and the fractional anisotropy value of molecular diffusion of the nerve roots could not be precisely measured; the shift point position identity of the DTI values was relatively poor.

- Our study may be one of the first studies to assess value of the unilateral lumbar disc prolapse in Egyptian patients; we found that DTI with fiber tracking is a non-invasive way to effectively trace the nerve fiber bundle and quantitatively evaluate the nerve injury and provide clinically relevant information and describe abnormalities beyond the resolution of conventional MR techniques and can be used for accurate diagnosis.

\section{Abbreviations}

FA: Fractional anisotropy; ADC: Apparent diffusion coefficient; JOA: Japanese Orthopedic Association; VAS: Visual analogue scale; DTI: Diffusion tensor imaging; DWI: Diffusion-weighted imaging; ROI: Region of interest; MRI: Magnetic resonance imaging

\section{Acknowledgements}

Not applicable.

\section{Authors' contributions}

AA wrote the manuscript and is responsible for correspondence to journal. MSh collected the patient data and participated in image processing and collection of patient's images. MH carried out the clinical assessment and performed the statistical analysis. AS participated in the study design and coordination and helped to draft the manuscript. All authors read and approved the final manuscript.

\section{Funding}

No funding sources.

\section{Availability of data and materials}

The datasets used and analyzed during the current study are available from the corresponding author on reasonable request.

\section{Ethics approval and consent to participate}

This study was approved by the medical ethical committee of "Faculty of Medicine Beni-Suef University" with ethical committee approval number FWA00015574. An informed written consent was taken from all subjects.

\section{Consent for publication}

All patients included in this research gave written informed consent to publish the data contained within this study.

\section{Competing interests}

The authors declare that they have no competing interests.

\section{Author details}

${ }^{1}$ Radiology Department, Faculty of Medicine, Beni-Suef University, Beni Suef, Egypt. ${ }^{2}$ Neurology Department, Faculty of Medicine, Beni-Suef University, Beni Suef, Egypt.
Received: 25 February 2020 Accepted: 6 December 2020

Published online: 13 January 2021

\section{References}

1. Boos N, Weissbach S, Rohrbach H et al (2002) Classification of age-related changes in lumbar intervertebral discs. Spine 27:2631-2644

2. Khalil C, Hancart C, Le Thuc V et al (2008) Diffusion tensor imaging and tractography of the median nerve in carpal tunnel syndrome: preliminary results. Eur Radiol 18(10):2283-2291

3. Kitamura M, Eguchi $Y$, Inoue $G$ et al (2012) A case of symptomatic extraforaminal lumbosacral stenosis ('far-out syndrome') diagnosed by diffusion tensor imaging. Spine 37:E854-E857

4. Mori S, van Zijl PC (2002) Fiber tracking: principles and strategies-a technical review. NMR Biomed 15:468-480

5. Hoy D, Brooks P, Blyth F et al (2010) The epidemiology of low back pain. Best Pract Res Clin Rheumatol 24(6):769-781

6. Dagenais S, Caro J, Haldeman S (2008) A systematic reviewof low back pain cost of illness studies in the United States and internationally. Spine J 8(1): 8-20

7. Papageorgiou AC, Croft PR, Ferry S et al (1995) Estimating the prevalence of low back pain in the general population. Evidence from the South Manchester back pain survey. Spine 20(17):1889-1894

8. Luoma K, Riihimaki H, Luukkonen R et al (2000) Low back pain in relation to lumbar disc degeneration. Spine 25:487-492

9. Cohen-Adad J, Benali H, Hoge RD et al (2008) In vivo DTI of the healthy and injured cat spinal cord at high spatial and angular resolution. Neuroimage 40(2):685-697

10. Li J, Cui H, Liu Z et al (2019) Utility of diffusion tensor imaging for guiding the treatment of lumbar disc herniation by percutaneous transforaminal endoscopic discectomy. Sci Rep 9:18753

11. Balbi V, Jean-François B, Duhamel A et al (2011) Tractography of lumbar roots: intial results. Eur Radiol 21:1153-1159. https://doi.org/10.1007/s00330010-2049-3

12. Shi $Y$, Zong $M, X u X$ et al (2015) Diffusion tensor imaging with quantitative evaluation and fiber tractography of lumbar nerve roots in sciatica. Eur J Radiol 84(4):690-695

13. Zhang J, Zhang F, Xiao F et al (2018) Quantitative evaluation of the compressed $\mathrm{L} 5$ and $\mathrm{S} 1$ nerve roots in unilateral lumbar disc herniation by using diffusion tensor imaging. Clin Neuroradiol 28(4):529-537

14. Eguchi Y, Ohtori S, Suzuki M et al (2016) Diagnosis of lumbar foraminal stenosis using diffusion tensor imaging. Asian Spine J 10(1):164-169

15. Eguchi Y, Ohtori S, Orita S et al (2011) Quantitative evaluation and visualization of lumbar foraminal nerve root entrapment by using diffusion tensor imaging : preliminary results. Am J Neuroradiol 32:1824-1829

16. Wu W, Liang J, Neng R et al (2016) Microstructural changes in compressed nerve roots are consistent with clinical symptoms and symptom duration in patients with lumbar disc herniation. Spine 41(11):E661-E666

17. Eguchi $Y$, Oikawa $Y$, Suzuki $M$ et al (2016) Diffusion tensor imaging of radiculopathy in patients with lumbar disc herniation. Bone Joint J 98-B: 387-394

18. Takashima H, Takebayashi T, Yoshimoto M et al (2013) Efficacy of diffusionweighted magnetic resonance imaging in diagnosing spinal root disorders in lumbar disc herniation. Spine 38(16):E998-E1002

19. Eguchi $Y$, Kanamoto H, Oikawa $Y$ et al (2017) Recent advances in magnetic resonance neuroimaging of lumbar nerve to clinical applications. Spine Surg Relat Res 1(2):61-71

20. Chen HB, Wan Q, Xu Q-F et al (2016) Reducing surgical levels by paraspinal mapping and diffusion tensor imaging techniques in lumbar spinal stenosis. J Orthopaedic Surg Res 11(1):47

\section{Publisher's Note}

Springer Nature remains neutral with regard to jurisdictional claims in published maps and institutional affiliations. 